\title{
Políticas sociales de protección a las familias en dificultad en tiempos de pandemia
}

\author{
Enrique Pastor Seller \\ Universidad de Murcia
}

Resumen

Las familias generan capital social para la sociedad, desempeñando un papel económico, social y político imprescindible para la cohesión y sostenibilidad social. La irrupción del coronavirus SARS-CoV-2 ha alterado profundamente las realidades familiares y las estrategias de cuidado. La pandemia ha evidenciado las debilidades de las políticas de protección social en general y las destinadas a las familias en particular. El artículo analiza los cambios en la estructura y comportamientos familiares, la exclusión social de los hogares y las políticas y programas de protección destinadas a las familias con mayores dificultades sociales que se desarrollan desde las diferentes administraciones públicas en España. La metodología de investigación se basa en la sistematización y análisis de fuentes estadísticas, estudios e investigaciones actualizadas y el análisis de contenido de las políticas, planes y medidas que se desarrollan desde los distintos niveles de administración pública, especialmente en el sistema de servicios sociales. Los resultados de la investigación constatan que las políticas sociales de protección y apoyo a las familias son escasas, insuficientes en cobertura y cuantía, fragmentadas y desiguales en los territorios. Es necesario incrementar la extensión de cobertura y la calidad de servicios y prestaciones sociales para mejorar la calidad y condiciones de vida de las familias, especialmente en tiempos de pandemia.

Palabras clave

Familias; Familias vulnerables; Protección social; Servicios Sociales; Políticas familiares

Códigos JEL: J18, I31, I38, Z18

\author{
SOCIAL POLICIES TO PROTECT FAMILIES IN DIFFICULTY IN \\ TIMES OF PANDEMIC
}

\begin{abstract}
Families generate social capital for society, playing an essential economic, social and political role for social cohesion and sustainability. The emergence of coronavirus SARS-CoV-2 has profoundly altered family realities and care strategies. The pandemic has highlighted the weaknesses of social protection policies in general and those aimed at families in particular. The article analyzes the changes in family structure and behavior, the social exclusion of households and the protection policies and programs aimed at families with greater social difficulties that are developed by the different public administrations in Spain. The research methodology is based on the systematization and analysis of statistical sources, updated studies and research, and the content analysis of the policies, plans and measures that are developed from the different levels of public administration, especially in the social services system. The research results confirm that social policies for the protection and support of families are scarce, insufficient in coverage and amount, fragmented and unequal in the territories. It is necessary to increase the extension of coverage and the quality of services and social benefits to improve the quality and living conditions of families, especially in times of pandemic.
\end{abstract}

Keywords

Families; Vulnerable families; Social protection; Social services; Family policies

JEL codes: J18, I31, I38, Z18

Fecha de recepción del original: 6 de febrero de 2021; versión definitiva: 15 de junio de 2021.

Enrique Pastor Seller, Departamento de Trabajo social y Servicios Sociales, Facultad de Trabajo Social, Universidad de Murcia, Campus de Espinarso, 30100 Murcia (España).

Tel.: +34 868884271; E-mail: epastor@um.es; ORCID ID: https://orcid.org/0000-0001-8693-5138. 


\section{Políticas sociales de protección a las familias en dificultad en tiempos de pandemia}

Enrique Pastor Seller

Universidad de Murcia

1. Introducción

La irrupción de la pandemia provocada por el coronavirus SARS-CoV-2 ha traído consigo incesantes y bruscas transformaciones sanitarias, sociales, relacionales, cotidianeidad, ocio, consumo, mercado de trabajo, económicas (micro y macro), hábitats, así como la propia percepción sobre las condiciones y calidad de vida. Sin duda un período de incertidumbres plenamente activo en la actualidad y cuyos efectos son todavía muy incipientes. La COVID-19 ha golpeado en un mundo que ya era profundamente desigual (Berkhout, et al., 2021), generando mayores desequilibrios territoriales y desigualdades sociales, económicas y digitales. Su impacto en España, según diferentes estudios (Oxfam-Intermón, 2021) nos podría devolver a índices de desigualdad semejantes a los experimentados durante los peores años de la crisis anterior, como resultado, sobre todo, de la pérdida de empleo de las personas más vulnerables: jóvenes, mujeres y migrantes, con efectos en niveles de pobreza inéditos.

España forma parte del grupo de países mediterráneos conocidos como «familistas» no precisamente por la atención ofertada a las familias desde el Estado sino por la contribución de éstas al entramado del bienestar en forma de servicios y cuidados (Moreno, 2013; Moreno y Vicente, 2016; Moreno, Ortega y Gamero, 2017). Las familias son fuente productora y beneficiaria de capital social para la sociedad (Marcaletti y Cavallotti, 2021), desempeñando un papel económico, social y político imprescindible para la cohesión y sostenibilidad social. Se sitúan en el centro de la vida social garantizando, junto a otros sistemas y actores, el bienestar de sus miembros, la educación y socialización de la infancia y la juventud y el cuidado de las personas mayores. Una institución ampliamente valorada por la ciudadanía, como señala el II Informe de Opiniones y actitudes sobre la familia (Centro de Investigaciones Sociológicas, 2014), el 98,7 \% de la población considera que la familia es algo "muy" o "bastante importante", situándose de forma abrumadora por encima de amigos, trabajo o dinero, des- tacando las funciones emocionales, crianza y educación.

El impacto de la pandemia ha alterado profundamente las estrategias familiares de cuidados. A pesar del avance que ha supuesto la puesta en marcha del Sistema para la Autonomía y Atención a la Dependencia [SAAD], su limitado desarrollo se manifiesta en la preponderancia de los cuidados informales desplegados tradicionalmente desde el entorno familiar (Asociación Estatal de Directores y Gerentes en Servicios Sociales, 2020). El cierre de muchos centros de día, comedores para mayores o la supresión o reducción de los servicios a domicilio y el miedo al contagio, ha supuesto un repliegue de la población mayor y dependiente en el hogar, un aumento de la carga de cuidados para las personas cuidadoras en el ámbito familiar, cuando estas existían, y un empeoramiento de las condiciones de vida de las personas dependientes, especialmente si vivían solas o en el caso cada vez más frecuente de parejas de personas de avanzada edad que se cuidan entre ellos/as. Hay que tener en cuenta el importante impacto en la salud y el deterioro de la autonomía personal que la soledad no deseada (De la Fuente, Martin y Ortega, 2019) y la pérdida de contacto social están teniendo en las personas dependientes que permanecen en sus hogares y han dejado de recibir servicios o no han logrado acceder a ellos (Consejo Económico y Social, 2021: 33).

Desde una perspectiva política, tener en cuenta a las familias supone un potencial para acelerar los logros de muchos de los objetivos de desarrollo sostenible [ODS]. Concretamente los relacionados con: promover el bienestar en todas las edades, eliminar la pobreza y el hambre; asegurar una vida sana; asegurar oportunidades educativas a lo largo de la vida y lograr la igualdad de género (Naciones Unidas, 2015). La Declaración de Copenhague de 2020 (Naciones Unidas, 2020a) reconoció a las familias, en su pluralidad, como la unidad básica de la sociedad, su papel clave en el desarrollo y, por tanto, el derecho a recibir protección y apoyo integral. Las políticas familiares orientadas a apoyar su papel cuidador, la conciliación laboral y familiar entre progenitores/tutores, la capacitación de las familias como actores de salud, educación y bienestar de sus miembros, han demostrado su capacidad para contribuir a los objetivos de desarrollo global, conforme se evidencia en los diferentes informes de seguimiento-evaluación de Desarrollo Sostenible (Naciones Unidas, 2020b). 
Familias nucleares, unipersonales, reconstituidas, monoparentales, homoparentales, monoparentales, multigeneracionales, etc. si bien todo este tipo de vinculaciones no constituyen en sí mismas una creación reciente, no deja de ser cierto que en las últimas décadas las familias han experimentado cambios sustanciales, tanto en su cuadro institucional como en sus composiciones, distribución territorial, funciones y expectativas. Del matrimonio concertado cuya función era la transmisión del patrimonio, pasando por la erróneamente denominada "familia tradicional", las familias adoptan una pléyade de expresiones "poliédricas" (Fundación ADECCO, 2019). En términos generales los patrones familiares son ahora más complejos y diversos por lo que los parámetros en torno a la noción de familia son más ambiguos (Bauman, 2001), lo que requiere de una amplia oferta de políticas públicas de atención y protección a las familias para su completa cobertura.

El articulo presenta los cambios en la estructura, actitudes y comportamientos familiares, acercándonos a las situaciones de dificultad y exclusión que afectan a los hogares, a partir de una metodología de investigación de carácter documental basada en el análisis de contenido de datos procedentes de fuentes estadísticas, estudios e investigaciones solventes en la materia en España. A continuación, se analizan las políticas y programas de bienestar social impulsadas por las diferentes administraciones públicas, con el fin de relacionar si éstas responden de manera coherente a las características y realidades de las unidades convivenciales actuales y futuras.

\section{Principales transformaciones socio-familiares.}

Las familias reflejan los cambios sociales y políticos, pero también son catalizadoras y actores básicos para impulsar las transformaciones sociales. Las estructuras familiares, las trayectorias de vida familiar y las relaciones intrafamiliares se encuentran inmersas en un profundo proceso de cambio. Según los últimos datos publicados por el Instituto Nacional de Estadística [INE] (INE, 2021a), España se situó en 47.351.567 habitantes a 1 de julio de 2020, con un aumento de 18.953 personas durante la primera mitad de año y un incremento anual relativo de 0,52 \%. Un aumento poblacional fruto de un saldo vegetativo negativo de 94.057 personas (167.559 nacimientos, frente a 261.616 defunciones), compensado por un saldo migratorio positivo de 113.856 .

De manera sintética destacar algunos datos sociodemográficos (INE, 2021a), que vislumbram los efectos de la COVID-19, por su incidencia en los cambios en las estructuras y dinámicas familiares: descenso de la tasa bruta de natalidad (-7.6 por mil) y de nupcialidad (-60,8 \%); aumento del número de parejas de hecho y nacimientos fuera del matrimonio; aumento de la edad media a la maternidad $(32,2 \%)$; incremento de matrimonios civiles; descenso de la fecundidad $(1,23)$; crecimiento de rupturas matrimo- niales; aumento de la proporción de matrimonios con al menos un cónyuge extranjero y del mismo sexo (subidas respectivas de $6,6 \%$ y 6,7 \%) - la mujer/madre sigue siendo quien ejerce la custodia en las separaciones (madre más del $76 \%$, padre: 5,57 \% y compartida: $18 \%$ ) -; incorporación masiva de las mujeres al mercado de trabajo con una importante y creciente brecha de género $-10,8 \%$, (INE, 2020a); aumento de la esperanza de vida (más de 83 años y superior en mujeres); envejecimiento de la población y aumento de proporción de personas mayores y dependientes. España, como evidencian las Proyecciones del INE 2020-2070 (INE, 2020b), es uno de los países que más se verá afectado por el envejecimiento de su población en los próximos años debido al importante volumen de su generación de "babyboomers", aunque los efectos de la pandemia probablemente modificaran el envejecimiento poblacional y la esperanza de vida.

La última Encuesta de Fecundidad de 2018 (INE, 2019), señala que el promedio de hijos/as deseado es 1,96 entre las mujeres y 1,86 entre los hombres. Parece evidente, por tanto, que los muy bajos niveles de fecundidad registrados en España no se derivan de un rechazo creciente de la maternidad o la paternidad, ni reflejan una preferencia mayoritaria por el hijo/a único/a. Por el contrario, la distancia que se observa entre la fecundidad deseada y la fecundidad alcanzada evidencia un déficit de bienestar individual y colectivo (Esping-Andersen, 2013) y sugiere la existencia de una serie de obstáculos que dificultan a muchas personas y parejas hacer realidad su proyecto familiar. El desempleo, la precariedad laboral, la incertidumbre sobre el futuro, el difícil equilibrio entre la vida laboral y familiar e insuficiente apoyo a la crianza y la desigualdad de género en la esfera familiar, son entre otros, algunos de los obstáculos al respecto (Castro, Martín, Cordero y Seiz, 2021).

3. "Nuevos" modelos convivenciales / familiares.

El modelo familiar español no es único, es cada vez más heterogéneo y reducido, emergiendo "nuevos modelos" familiares. En Encuesta Continua de Hogares [ECH] (INE, $2021 \mathrm{~b}$ ), observamos el incremento constante de hogares, alcanzando 18.754 .800 (2020), con un incremento anual de $129.100(0,7 \%)$.

La población residente en viviendas familiares se incrementó un 0,5\%, mientras que el tamaño medio de los hogares se mantuvo en 2,50 personas por hogar. Los hogares más frecuentes en 2020 volvieron a ser los formados por dos personas (30,4\% del total). Le siguieron los unipersonales $(26,1 \%$, aunque la población incluida en estos sólo supuso el 10,4\% del total). Por su parte, los hogares de cinco o más personas constituyeron el 5,8 \% del total. Su tamaño medio fue de 5,60 personas y concentraron al 13,1\% de la población. Por tamaño, el número de hogares de una persona aumentó un 2,0 \% en 2020, el de dos un 0,6 \% y el 
de cinco o más personas creció un 1,3\%. Por el contrario, el número de hogares con tres y cuatro personas se redujo un $0,1 \%$ y un $0,4 \%$, respectivamente, respecto a 2019. Según las proyecciones de hogares 2020-2035 (INE, 2020b) los hogares unipersonales se incrementarán en dicho periodo en 1.103.761, alcanzando la cifra de 19.796 .040 (5,9\%), reduciéndose el tamaño medio del hogar que pasaría de los 2,51 actuales a 2,41 personas por hogar en 2035. Datos que constatan la tendencia a una progresiva disminución en el tamaño de hogares y un aumento en su número y tipología.

La familia nuclear (matrimonio con o sin descendencia) es la fórmula mayoritaria de convivencia, aunque va disminuyendo año tras año. A su vez, es cada vez menos frecuente la presencia de varios hijos/as. La mayor parte son los formados por parejas con hijos $(33,1 \%)$ y sin hijos representan el 20,9\%.

En cuanto a los hogares unipersonales, había 4.849.900 personas viviendo solas (26,1\%) en el año 2020 , con un incremento anual de 96.200 (2\%). De esta cifra, 2.131.400 $(43,6 \%)$ tenían 65 o más años, y de ellas, $1.511 .000(70,9$ $\%)$ eran mujeres. El porcentaje de personas que viven solas difiere por sexo y edad. Así, en las edades hasta 64 años es más elevado en los hombres $(59,1 \%)$, mientras que en las edades mayores de 65 años es mayor en las mujeres.

Respecto al estado civil, los hogares unipersonales de hombres más frecuentes están formados por solteros $(59,7$ $\%)$ y los de mujeres por viudas (45,5\%). Por edad, el 44,1 $\%$ de las mujeres mayores de 85 años viven solas, frente al $24,2 \%$ de los hombres. Estos datos, revelan que los patrones clásicos de convivencia están cambiando, incrementándose el número de hogares unipersonales especialmente entre las personas mayores, siendo este hecho más notable entre las mujeres, en consonancia con otras investigaciones (Alaminos y Ayuso, 2019).

Según la ECH (INE, 2021b), los hogares monoparentales/monomarentales se han incrementado superando los 1.944 millones (10,4 \% de hogares), con un aumento de 57.300 (3\%), en consonancia con la tendencia de los últimos años. Una característica relevante de este tipo de hogar es que está fuertemente feminizado: un $81,4 \%$ del total está encabezado por una mujer $(1.582 .100$, con un incremento del 3,4\%), frente a los 362.700 (1,6\%) por padre; de ahí que se prefiera utilizar el término "monomarentales",

El número de hogares monoparentales/monomarentales ha aumentado al crecer el número de rupturas, separaciones y divorcios, por un lado, pero también por el incremento de la esperanza de vida particularmente de las mujeres (viudedad). Por otra parte, el número de mujeres que afrontan la maternidad a solas también ha crecido, no tanto por las causas tradicionales (embarazo no planificado en solitario), sino por decisión propia (embarazo planificado, subrogación y/o adopción), con independencia de la existencia de un vínculo conyugal con el progenitor. Así, el origen de estos hogares monomarentales atiende a realidades muy diferentes, según la Fundación ADECCO (2019): la elección de asumir en solitario la maternidad (41,3\%), ruptura $(34,4 \%)$, fallecimiento $(1,3 \%)$ u otro tipo de situaciones como la migración. El 68,2 \% de los hogares monoparentales está compuesto por dos miembros y sólo en un $27,2 \%$ por tres personas. A pesar de esta diferenciada composición de los hogares monoparentales, como veremos más adelante, las principales políticas y medidas están referidas al porcentaje minoritario, cuando el problema no es tanto el número de hijos/as, como la presencia de una única persona adulta sustentadora.

Según la ECH (INE, 2021b), se constata un aumento de las familias reconstituidas tanto heterosexuales como homosexuales, siendo a su vez muy plurales. Los datos revelan que un $14 \%$ de las familias españolas son reconstituidas, lo que evidencia su aumento progresivo. Según el VII Informe sobre exclusión y desarrollo social en España (Castro y Seiz, 2014) y otros estudios al respecto (Verde, González, Vázquez y Moreno, 2020), en aproximadamente uno de cada cinco matrimonios ( $22 \%$ ) es un segundo matrimonio para al menos uno de los cónyuges, y esta cifra solo capta parcialmente la incidencia de segundas uniones, ya que las personas que han experimentado una ruptura conyugal son más propensas a optar por una pareja de hecho que un matrimonio como segunda unión.

En cuanto a la emancipación juvenil residencial, el 55,0 $\%$ de los jóvenes entre 25 y 29 años vivía con sus padres o con alguno de ellos en 2020. Este porcentaje ha crecido 6,5 puntos en los siete últimos años. Por sexo, el porcentaje de no emancipados fue del $62,9 \%$ en los hombres y del 46,9 $\%$ en las mujeres. Por su parte, el porcentaje de personas de 30 a 34 años que vivían con sus padres o con alguno de ellos se situó en el 25,6\%, con un aumento de 5,1 puntos desde 2013. En los hombres el porcentaje fue del 31,3 \% y en las mujeres del $20,0 \%$. Estos datos evidencian la progresiva reducción de la emancipación juvenil (Moreno, López y Segado, 2012; Consejo de la Juventud, 2020a), situándonos como señalan Donat y Martín-Lagos (2020) en cifras similares a otros países del sur de Europa. La desigualdad en las transiciones hacia la vida adulta se refleja en la desproporción en el peso relativo de los hogares unipersonales: en el segundo trimestre de 2020 , tan solo el $12,9 \%$ de las mujeres jóvenes emancipadas vivía sola, mientras que prácticamente uno de cada cuatro hombres jóvenes lo había conseguido (24,8 \%).

La crisis que acompaña a la pandemia de la COVID-19 ha incidido también en el comportamiento residencial de la población joven (Consejo de la Juventud, 2020b), aunque sus consecuencias sobre el empleo han sido todavía mayores. La población joven se encuentra especialmente afectada por: menos empleo, más estacionalidad, descenso de la ocupación, ocupación en situaciones de riesgo - ERTE o similar - y refugio en estudios ante las escasas expectativas laborales. En la actualidad, aproximadamente un tercio de la población de entre 16 y 29 años está empleada, el $66 \%$ restante, se reparte entre población inactiva $(52,1 \%)$ y población en paro (14,4 \%) (López Oller, 2020). A ello debemos añadir, entre otras, las dificultades de comprar/alquilar una vivienda, de ahí que son cada vez más las personas jóvenes que comparten viviendas de alquiler. A pesar de la progresiva normalización en nuestra sociedad de la emancipación en pisos compartidos, la emancipación en solitario sigue siendo un reto, dado que solo el $16 \%$ del total de la población joven emancipada vive sola. Como señalan Donat, Martín y Moles (2020:12), seguir en el hogar de los progenitores, por un lado, permite a los jóvenes enfrentarse a las dificultades del mercado de trabajo y, por 
Cuadro 1. Tipo de Unidad de Convivencia de personas titulares de Rentas Mínimas de Inserción

\begin{tabular}{|c|c|c|c|c|c|c|}
\hline \multirow{2}{*}{ Unidad Convivencia } & \multicolumn{2}{|c|}{ Mujeres } & \multicolumn{2}{|c|}{ Hombres } & \multicolumn{2}{|c|}{ Total } \\
\hline & No & $\%$ & No & $\%$ & No & $\%$ \\
\hline Unipersonales & 46.762 & 43,5 & 60.865 & 56,6 & 107.627 & 46,7 \\
\hline Monoparentales & 52.714 & 93,6 & 3.583 & 6,4 & 56.297 & 24,4 \\
\hline Biparentales & 27.632 & 61,8 & 17.106 & 38,2 & 44.738 & 19,4 \\
\hline Pareja sin hijos/as & 5.426 & 56,5 & 4.182 & 43,5 & 9.608 & 4,2 \\
\hline Otros & 7.337 & 60,4 & 4.811 & 39,3 & 12.148 & 5,2 \\
\hline Total & 139.871 & 60,7 & 90.547 & 39,3 & 230.418 & 100,0 \\
\hline
\end{tabular}

otro, les posibilita seguir formándose o mantenerse en la búsqueda un empleo que concuerde con sus capacidades y formación.

4. Familias en situación de dificultad social.

Según la última Encuesta de Condiciones de Vida [ECV] de 2019 (INE, 2020C), la población en riesgo de pobreza o exclusión social (AROPE) se situó en el 25,3\%, frente al 26,1 $\%$ (2018) y 26,6 \% (2017). La reducción se produce en dos de sus tres componentes: carencia material severa (del 5,4 $\%$ al $4,7 \%$ ) y riesgo de pobreza (del $21,5 \%$ al 20,7 \%), mientras que la baja intensidad en el empleo subió una décima y se situó en el 10,8\%.

Considerando dicha tasa y atendiendo al tipo de hogar, se sitúan por encima de esa media las personas que vivían en hogares formados por un/a adulto/a con 1 o más hijos/as dependientes a su cargo $(46,8 \%)$, otros hogares con niños/as dependientes $(33,4 \%)$ y hogares de una persona (26,1\%). Por debajo de la media, dos adultos con 1 niño/a o más dependiente/s (25,2 \%), otros hogares sin niños/as dependientes (21.79) y dos adultos sin niños/as dependientes (20,3\%). El análisis longitudinal (2009-2019), vuelve a evidenciar la difícil situación de las personas solas adultas que viven en hogares con niños/s a su cargo, cuyas tasas AROPE son hasta 27 puntos más elevadas que los hogares compuestos sólo por personas adultas. Dentro de ellos destaca la situación de los hogares monoparentales, cuya tasa AROPE ha sido extraordinariamente alta durante todo el período estudiado sin apenas mejorar (2009: 51,1 $\%$ y $2019: 46,8 \%)$.

Los hogares monomarentales por sus características intrínsecas están especialmente sobrerrepresentados en la exclusión social, afectando fundamentalmente a la mujer (feminización de la monoparentalidad). En su relación con el empleo, aquellas personas a cargo de uno/a o más niños/as suelen encontrar mayores dificultades y barreras a la hora de ascender o emprender una carrera profesional, y suelen acceder a jornadas parciales o reducidas por falta de medidas de conciliación y la carga de tareas. Así, la relación entre pobreza, exclusión social y hogares monomarentales es clara ya que estos hogares están expuestos a mayores factores de vulnerabilidad y desigualdad (Moreno, 2000; Sastre, 2015; Almeda, Vergés, Obiol y Moreno, 2016; Fundación FOESSA, 2019).

Los hogares monoparentales, encabezados mayoritariamente por mujeres, experimentan las tasas de riesgo de pobreza relativa más altas por tipo de hogar, pasando del $36,8 \%$ (2008) al 42,9 \% (2018). Según el $9 \circ$ Informe de parentalidad y empleo (Fundación ADECCO, 2020), el $80 \%$ de las mujeres que lideran familias monoparentales ha visto empeorar su situación laboral y económica con la pandemia.

La tasa de riesgo de pobreza de niños/as que viven en hogares monoparentales es sistemáticamente superior a la tasa media de pobreza infantil. Aunque desde 2011 las diferencias se están reduciendo, aún ahora se mantiene en tasas unos 15 puntos superior a la media (Malgesini, 2019: 45-46). La estructura de los hogares en los que viven los menores en España presenta diferencias importantes en función del origen inmigrante o autóctono de sus padres. El análisis longitudinal (2008-2017) evidencia que las mayores tasas de pobreza entre la población infantil se encuentran con padres inmigrantes todos los tipos de hogar, es decir, la tasa de pobreza infantil es siempre mayor entre menores con ambos progenitores nacidos fuera de España (Ministerio de Sanidad, Consumo y Bienestar Social, 2019b: 29).

Para complementar este análisis, cabe analizar el perfil de la persona perceptora de rentas mínimas autonómicas en España, conforme a cuadro siguiente, caracterizado por ser: mujer española, entre 35 y 44 años, con hijos/as a cargo y bajo nivel de estudios. El 60,70 \% de las personas perceptoras son mujeres, mayoritarias en todos los tipos de hogar a excepción de los unipersonales. El 93,6 \% de los hogares monoparentales perceptores está encabezado por mujeres. 
Los datos provisionales referidos a 2019 (Ministerio de Derechos Sociales y Agenda 2030, 2020a) señalan que el $43,40 \%$ de los hogares son familias unipersonales, el 21,83 $\%$ monoparentales, un 21,09 \% biparentales, 6,54 \%, parejas sin hijos y un 7,51\% otras modalidades de hogares. Respecto al tipo de hogar por número de miembros con los que cuenta la unidad de convivencia, 33,47 \% son unipersonales, $19,76 \%$ hogares de dos miembros, 13,46\% de tres miembros, 9,04\% de cuatro miembros, 5,58 \% de cinco miembros, 2,23\% de seis miembros, 0,68\% de siete miembros y $0,25 \%$ de 8 o más miembros. Diferentes estudios (Autoridad Independiente de Responsabilidad Fiscal, 2019; FOESSA, 2019), constatan que los hogares unipersonales y monoparentales muestran una mayor probabilidad de sufrir dependencia respecto de los servicios sociales públicos con respecto a los que viven en pareja.

El análisis de los datos muestra al igual que otros estudios (Pastor et al., 2020; González y Pastor, 2019; OxfamIntermón, 2019; Pastor, Verde y Lima, 2019) la evidente feminización de la pobreza, a la vez, que son las mujeres las que siguen siendo quienes se ocupan, principalmente, de las labores del "cuidado" y de la asistencia a sus miembros, especialmente ante situaciones de dependencia. La demanda en el Sistema Público de Servicios Sociales se formaliza a través del indicador de: "mujer" aunque el análisis de la situación problema alude a "familias con necesidad de intervención social".

5. Planes y estrategias sociales internacionales y estatales en tiempos de pandemia.

La Carta de Derechos Fundamentales de la Unión Europea en su capítulo IV Solidaridad, dedica el articulo 33 a la "vida familiar y vida profesional" señalando la garantiza a la protección de la familia en los plano jurídico, económico y social (art. 33.1). En sintonía, la Constitución Española de 1978 (España, 1978) responsabiliza a los poderes públicos aseguran la protección social, económica y jurídica de la familia (arts. 9 y 39.1). Resulta relevante aludir a la legislación recientemente aprobada respecto a la ampliación de los derechos de paternidad (Jefatura del Estado, 2019) - que modifica la Ley Orgánica 3/2007, de 22 de marzo - y la Ley Orgánica 8/2021, de 4 de junio, de protección integral a la infancia y la adolescencia frente a la violencia (Jefatura del Estado, 2021a)

En el Informe sobre la aplicación de los objetivos del Año Internacional de la Familia y sus procesos de seguimiento (Naciones Unidas, 2019a), se examinan las tendencias en políticas familiares (protección social, conciliación, igualdad, sin hogar) y buenas prácticas en su diseño.

Con el fin de ayudar a reparar los daños económicos y sociales causados por la pandemia del coronavirus, la Comisión Europea, el Parlamento Europeo (2020) y el Consejo alcanzaron el 18 de diciembre de 2020 un Acuerdo sobre el Mecanismo de Recuperación y Resiliencia, instrumento que ocupa un lugar central en Next Generation EU. Un Fondo de Recuperación y Resiliencia sin precedentes orientado a impulsar el proceso de reconstrucción de las economías en el mundo post-pandemia, a partir de 2021 (Comisión Europea, 2020). Sus recomendaciones y plan de financiación se encuentran en la base del Plan de Recuperación, Transformación y Resilencia, aprobado por el Gobierno de España, mediante Real Decreto-Ley 36/2020, convalidado el 28 de enero de 2021 por el Congreso de los Diputados (Cortes Generales, 2021). El Plan de recuperación, transformación y resiliencia (Presidencia del Gobierno, 2021) pretende contribuir de manera sustantiva a reparar los daños económicos y sociales causados por la pandemia del coronavirus. Contempla diez "políticas palanca", destacando la VIII relativa a la "nueva economía de los cuidados y políticas de empleo" y, concretamente su componente 22: Plan de choque para la economía de los cuidados y refuerzo de las políticas de inclusión. De esta forma, el refuerzo y la modernización de las políticas de cuidados, en particular y de los servicios sociales en general, es uno de los elementos estratégicos.

La administración central asume las políticas de familias e infancia, fundamentalmente, a través de la Secretaria de Estado de Derechos Sociales del Ministerio de Derechos Sociales y Agenda 2030 (Ministerio de Política Territorial y Función Pública, 2020) concretamente: la protección y promoción de las familias y su diversidad (art. 2.2.c), la infancia y la adolescencia (art. 2.2.d), a través de la Dirección General de Diversidad Familiar y Servicios Sociales y de Derechos de la Infancia y de la Adolescencia, respectivamente. El último Plan de Apoyo a las Familias de España aprobado (Ministerio de Sanidad, Consumo y Bienestar Social, 2015), recoge un total de 228 medidas que se articulan en torno a 7 líneas estratégicas: protección social y económica de las familias en situaciones en dificultad social; conciliación y corresponsabilidad; apoyo a la maternidad y entorno favorable a la vida familiar; parentalidad positiva; apoyo a familias con necesidades especiales, coordinación institucional y evaluación transversal.

Por su parte, la Estrategia Nacional de Prevención y Lucha contra la Pobreza y la Exclusión Social 2019-2023 (Ministerio de Sanidad, Consumo y Bienestar Social, 2019b) aborda de forma transversal dos asuntos fundamentales como son la protección de la infancia y la protección de las familias, actuando especialmente en situaciones de mayor vulnerabilidad. Estos se articulan en torno a cuatro metas estratégicas: combatir la pobreza; generar inversión social, consolidar un sistema de servicios públicos sanitarios, educativos y sociales universales y promover la eficacia y eficiencia de las políticas a través de la coordinación interinstitucional y la cogobernanza.

De manera concreta, la estrategia orientada a consolidar un sistema de servicios públicos sanitarios, educativos y sociales universales contempla dos objetivos: "apoyo a menores y familias" (3.2) y "servicios sociales y dependencia" (3.3). El primero pretende promover una crianza positiva y saludable para todas las familias, desarrollando políticas favorables a las familias y facilitando apoyos desde los servicios sociales, sanitarios y educativos a los colectivos 
vulnerables. El segundo, orientado a mejorar la calidad de vida y el pleno desarrollo de las personas y unidades familiares más vulnerables y atender las necesidades derivadas de las situaciones de exclusión social y dependencia.

En sintonía con las medidas de lucha contra la pobreza mencionadas y los efectos sociales derivados de la COVID-19, la administración central ha impulsado conjunto de medidas y prestaciones sociales y económicas dirigidas a familias y colectivos vulnerables en el ámbito de la vivienda (suspensión de desahucios y lanzamientos, moratoria rentas, prorroga contratos de arrendamiento, ayudas al alquiler), garantía de suministro de energía eléctrica y otros productos derivados, proteger el empleo, ampliar los subsidios, protección de consumidores, protección a mujeres que sufren violencia de género, becas de comedor, fondo para apoyo a familias en tareas de cuidados, entre otros (Jefatura del Estado, 2020a; 2020b; 2020c; 2021 b).

El IMV (Jefatura del Estado, 2020d), es impulsado como una prestación dirigida a prevenir el riesgo de pobreza y "amortiguar" la exclusión social de las personas que viven solas o están integradas en una unidad de convivencia y carecen de recursos económicos básicos para cubrir sus necesidades básicas. En la reciente revisión normativa de la prestación (Jefatura del Estado, 2021c), se elimina el límite de titulares del ingreso mínimo vital en una misma vivienda, con el fin de eliminar las barreras de acceso al mismo que han podido experimentar las personas usuarias de prestaciones de servicio residencial, así como de reconocer la realidad de personas en situación de vulnerabilidad que se agrupan en una misma vivienda con el fin exclusivo de compartir gastos. Se configura como derecho subjetivo a una prestación económica, que forma parte de la acción protectora de la Seguridad Social, y garantiza un nivel mínimo de renta a quienes se encuentren en situación de vulnerabilidad económica. Persigue garantizar una mejora real de oportunidades de inclusión social y laboral de las personas beneficiarias. La implantación del IMV está teniendo incidencia sobre el conjunto de rentas mínimas de las comunidades autónomas. Destacar que las familias monomarentales y otros colectivos tienen protecciones adicionales

Con un $27,4 \%$ de los niños y niñas en riesgo de pobreza en España, el Alto Comisionado contra la Pobreza Infantil (2021), ha lanzado la iniciativa "Alianza País Pobreza Infantil Cero", que tiene como fin cambiar la senda de la pobreza infantil mediante el fomento de la participación y la consecución de acuerdos con más de 75 actores clave (administración púbica, empresas y fundaciones y tercer sector) para conseguir un país que proporcione iguales oportunidades a todos sus ciudadanos y romper el círculo de la pobreza infantil. Nace dos metas: garantizar una educación inclusiva y de calidad y generar entornos saludables, positivos y seguros.

Además de lo anteriormente expuesto, siguen existiendo programas y prestaciones destinadas a familias (Ministerio de Derechos Sociales y Agenda 2030, 2021) que se impulsan desde diferentes organismos públicos (Seguridad Social, Agencia Tributaria, Servicio Público de Empleo y Formación, etc.) o privados (empresas eléctricas, etc.).
6. Medidas y prestaciones de protección social desde las administraciones autonómicas y locales.

Las Comunidades Autónomas, ejercen las competencias de promoción, protección y apoyo a las familias e infancia, elaboración de planes, programas y proyectos específicos, protección y tutela de menores, desarrollo de programas para la preparación a la vida independiente, adopción y parejas de hecho, entre otras.

Las competencias en materia de Servicios Sociales en España se encuentran descentralizadas en las diferentes Comunidades Autónomas y Corporaciones Locales, atendiendo a sus respectivos Estatutos de Autonomía, Leyes de Servicios Sociales de las Comunidades Autónomas, Leyes de Infancia autonómicas y la Ley de Bases de Régimen Local. Por otra, los Servicios Sociales especializados en materia de infancia articulan la oferta y procedimientos de intervención, impulsando diferentes programas de intervención familiar y de defensa de los derechos de los niños, las niñas y adolescentes.

Los proyectos sociales de protección a la familia y atención a la pobreza infantil desarrollados en las Comunidades Autónomas y Ciudades Autónomas en colaboración con las Corporaciones Locales son (Ministerio de Derechos Sociales y Agenda 2030, 2020b), fundamentalmente los siguientes proyectos: paliar y mejorar la situación de vulnerabilidad social de las familias; apoyo a la conciliación familiar y laboral y servicios de intervención y apoyo familiar, entre los que se encuentran los definidos en el apartado 1.3, del Catálogo de referencia de Servicios Sociales (Ministerio de Sanidad, Consumo y Bienestar Social, 2013: 28).

Los servicios sociales de atención primaria ofrecen atención a las familias a través de las siguientes prestaciones:

- Información, orientación, valoración, diagnóstico y asesoramiento.

- Prevención de situaciones de desprotección y conflictos social en el ámbito familiar.

- Atenciones de situaciones de emergencia social por catástrofes naturales o situaciones de emergencia derivadas de conflictos familiares y sociales.

- Intervención social, psicológica y educativa con familias en riesgo social.

- Ayuda a domicilio y apoyo a la unidad convivencial.

- Alojamiento temporal o permanente alternativo a las personas carentes de hogar o con graves problemas de convivencia.

- Prevención e inserción social.

- Fomento de la solidaridad y cooperaciones a través de acciones dirigidas a toda la población para promover actitudes favorecedoras de la convivencia ciudadana, la promoción del voluntariado y de la participación.

Los servicios sociales municipales impulsan proyectos que ofrecen respuestas a necesidades sociales de distintas áreas, promoviendo intervenciones interdisciplinarias para 
la prevención, y atención de familias que se encuentren en situación de riesgo social, para impulsar una atención temprana y proporcionar los apoyos necesarios o, en su caso, proceder a su derivación a los servicios sociales especializados (Pastor y Sánchez, 2020).

En Colaboración con entidades locales y tercer sector destacar las actividades de intervención comunitaria, familiar en Corporaciones Locales (PRAS); mantenimiento y programación de actividades a Asociaciones sin ánimo de lucro de familiares, amigos y enfermos drogodependientes; mantenimiento de centros, servicios y desarrollo de programas de actividades destinadas a infancia, familia y menores afectados por medidas de protección y/o judiciales.

\section{Conclusiones y propuestas.}

Las familias, en consonancia con las dinámicas sociales, adoptan expresiones muy diversas, con cambios constantes en su estructura, composición, funciones, actitudes y expectativas. Los nuevos modelos convivenciales, la feminización de la pobreza, las desigualdades de género, el envejecimiento de la población, las crecientes tasas de dependencia, el descenso de la población en edad de trabajar y el número de mujeres en edad fértil, entre otros, plantean la necesidad de abordar el diseño y sostenibilidad de políticas sociales orientadas a la atención y protección de las familias en general $y$, en dificultad social en particular en España. El análisis del diseño de las políticas familiares debe tener en cuenta la heterogénea composición de los distintos hogares/unidades convivenciales, su desigual distribución territorial desigual, con el fin de que ofrecer respuestas a realidades y necesidades concretas y asimétricas en las diferentes Comunidades y Ciudades Autónomas, e incluso en el seno de estas regiones, provincias y municipios, de ahí la importancia de las políticas autonómicas y locales.

Como se ha analizado en el texto, en España las políticas de apoyo a las familias son muy escasas, insuficientes en cobertura y cuantía, fragmentadas y desiguales en los territorios. Las políticas sociales dirigidas a las familias y los modelos familiares apoyados públicamente dan forma a la estructura de oportunidades que encuentran las mujeres. Las familias todavía actúan como amortiguadores sociales y proveedores de recursos, dentro de esquemas de políticas de protección social basadas en el principio de subsidiariedad. Las políticas tienen, además, un carácter simbólico, porque se basan en determinados modelos familiares y de género. Si las políticas públicas parten de atribuir los cuidados a uno de los dos cónyuges en detrimento del otro, esto reforzará y legitimará una división del trabajo tradicional y asimetría. De hecho, los diseños institucionales suelen albergar ideas implícitas sobre la división de los cuidados según género. Por ello, las políticas familiares deban diseñarse desde la perspectiva de género, siendo además una recomendación internacional (Naciones Unidas, 2019b)

Las investigaciones muestran que las parejas suelen separar sus ideales de dedicación al trabajo y al cuidado de sus posibilidades reales. Los padres y madres acaban desarrollando "preferencias adaptativas» para hacer frente a los desequilibrios entre sus ideales sobre el cuidado y las limitaciones a las que se enfrentan. Los comportamientos ante las políticas sugieren una construcción de género diferencial que no necesariamente - o no exclusivamentetiene que ver con condicionamientos laborales o consideraciones económicas.

Las familias monomarentales constituyen un fenómeno creciente en nuestras sociedades, generando nuevos retos para las políticas públicas porque cuestionan pilares sobre los que se ha sustentado el modelo familiar $y$, a su vez, demandan nuevas políticas familiares que respondan a su diversidad y necesidades intrínsecas (Almeda, Vergés, Obiol y Moreno, 2016). Conforme hemos podido evidenciar del análisis realizado, las políticas públicas no están atendiendo de manera eficaz a la diversidad de estas familias, así como a las reconstituidas. Las familias monomarentales, dada sus especiales dificultades, precisan de una protección especial, ya que sus integrantes adultos y niños/ as sufren, como hemos analizado anteriormente, mayores tasas de pobreza y exclusión social. Por ello, la primera recomendación, como señalan diferentes autoras y estudios (Malgesini, 2019) es sancionar una legislación específica, de ámbito estatal y autonómico, sobre hogares monomarentales a fin de garantizar derechos, eliminar la discrecionalidad y las prácticas discriminatorias. La emergencia sanitaria ha destapado como nunca las dificultades que afrontan los hogares sustentados por un solo progenitor, reavivando la reivindicación histórica del establecimiento de un marco regulador específico que dote de protección a las familias monoparentales

Invertir en políticas centradas en la familia es importante para luchar contra la pobreza, la desigualdad y la exclusión social. El análisis de las políticas familiares internacionales (Naciones Unidas, 2019a) identifica algunas líneas de acción. Los programas de transferencias monetarias y ayudas a la vivienda a familias en situación de vulnerabilidad son una buena estrategia para prevenir la pobreza y la falta de hogar. La conciliación de la vida laboral y familiar de las familias con hijos y la redistribución de los cuidados en el hogar ayudan a los progenitores a conciliar sus obligaciones laborales y familiares. Para ello la concesión de incentivos a empresas y el derecho a licencia parental permiten aumentar la integración laboral de las mujeres. Es imprescindible reforzar las acciones de concienciación y educación para la igualdad de oportunidades, de género mediante programas y acciones de proximidad local (formación en género y derechos, programas de parentalidad positiva, mediación familiar, simplificar reclamación de pensiones de alimentos, etc.).

El IMV ha tenido, hasta la actualidad, un impacto limitado en la reducción de la desigualdad en España. A las dificultades de su implementación se añade el bajo porcentaje de potenciales beneficiarios/as que han podido acceder a este tipo de protección. Según estimaciones del Ministe- 
rio de Inclusión Social y Seguridad Social, hasta mediados de diciembre de 2020 solo alcanza a 159.500 hogares, el $20 \%$ de los 850.000 previstos. La lenta gestión del ingreso mínimo vital coincide con un descenso en la cobertura que proporcionan las rentas autonómicas, con una importante reducción respecto a años anteriores (Diario Público, 2021). De haberse desarrollado en su totalidad durante el segundo semestre del año, el IMV habría reducido en 0,5 puntos el índice de Gini, salvando de la pobreza a 277.000 personas, y reduciendo la pobreza severa en 230.000 personas. En medio de una situación económica y sanitaria extraordinariamente grave, no se está consiguiendo dar respuesta y solución, con la urgencia y necesidad requerida, a los hogares más vulnerables, ahogados en una burocracia administrativa que dificulta que las ayudas lleguen a tiempo y en un contexto donde están cerradas muchas posibilidades de encontrar un empleo. La nueva regulación (Jefatura del Estado, 2021b) permite que entidades del tercer sector de acción social inscritas como"mediadores sociales del IMV" emitan certificados para acreditar situaciones de convivencia complejas, lo que ha sido criticado por sindicatos (CC.OO. y UGT). Amplia el límite de titulares de una misma vivienda e introduce el concepto de unidad de convivencia independiente (familia nuclear) en casos de mujeres víctimas de violencia de género, divorcio o separación, así como afectados por desahucio o inhabitabilidad para permitir el acceso a la prestación. La liberación de recursos que posibilita la implantación de la nueva prestación abre el debate sobre el destino de los mismos, que no debe ser un mero ahorro de gasto social autonómico por sustitución del gasto social del Estado. Es necesaria la adaptación de las distintas rentas de las CCAA con el IMV, con un resultado final de aumento del gasto social para una necesidad que, en tiempos de pandemia, además, está incrementándose exponencialmente. Por otra parte, se considera necesario agilizar el proceso de reconocimiento del IMV y la flexibilización de los requisitos, facilitando la gestión tanto a los demandantes, como a las personas que tramitan los expedientes, incrementando las plantillas y simplificando el sistema de gestión.

Las familias continúan actuando como principal amortiguador de los efectos que se derivan en situaciones de crisis sanitarias, económicas y sociales, complementando el papel que desempeñan las políticas públicas, en la redistribución de rentas entre generaciones y la atención a personas con mayores dificultades. Esta situación la volvemos a encontrar en la actual situación de pandemia derivada de la COVID-19, donde se evidencia que las familiares se encuentran especialmente afectadas tanto en sus indicadores objetivos como subjetivos (Espinosa y Pérez, 2021; Fundación The Family Watch, 2021). La pandemia ha puesto de manifiesto la importancia de invertir en políticas sociales que protejan a las personas y familias más vulnerables. Son las familias quienes se llevan la peor parte de la crisis, se ven obligadas a proteger a sus miembros, cuidar de sus hijos que no pueden asistir a la escuela y continuar con sus responsabilidades laborales. Las familias se encuentran en el centro de las interacciones intergeneracionales y están siendo un actor de apoyo social en esta crisis, pero es necesario cuidar a las familias, especialmente más vulnerables. Los vertiginosos cambios y tendencias familiares anali- zadas evidencian, en sintonía con otros estudios sobre la materia (López y Castillo, 2020), la necesidad de un diseño dinámico y sostenible de las políticas e intervenciones sociales (López y Segado, 2013; Pastor, 2017) de apoyo a las familias en España en sintonía con ellos.

Bibliografía

ALAMINOS, E. y AYUSO, M. (2019): “Estado civil, género, mortalidad y pensiones: las desventajas de la soltería en la vejez", Revista Española de Investigaciones Sociológicas, 165, pp. 3-24.

ALMEDA, E.; VERGÉS, N.; OBIOL, S. y MORENO, A. (2016): “Monoparentalidades y políticas públicas", ARXIUS, 34, pp. 43-58.

ALTO COMISIONADO CONTRA LA POBREZA INFANTIL (2021): Alianza País Pobreza Infantil Cero. Documento de trabajo. Madrid, Alto Comisionado contra la Pobreza Infantil. Disponible en https://www.comisionadopobrezainfantil.gob.es/sites/default/files/Documento \%20de \%20trabajo \%20 \%27Alianza \%20Pa \%C3 \%ADs \%20Pobreza \%20 Infantil \%20Cero \%27.pdf (Consultado 4/02/2021).

ASOCIACIÓN ESTATAL DE DIRECTORES Y GERENTES EN SERVICIOS SOCIALES (2020): XX Dictamen del Observatorio de la Dependencia. Disponible en https://dependencia.info/imagenes/xx-dictamenobservatorio-dependencia.pdf (Consultado 10/12/2020).

AUTORIDAD INDEPENDIENTE DE RESPONSABILIDAD FISCAL [AIReF] (2019): Los programas de rentas mínimas de España. Madrid, Autoridad Independiente de Responsabilidad Fiscal. Disponible en https:// www.airef.es/wp-content/uploads/RENTA_MINIMA/20190626-ESTUDIO-Rentas-minimas.pdf (Consultado 15/10/2020).

BAUMAN, Z. (2001): La sociedad individualizada. Madrid, Cátedra.

BERKHOUT, E.; GALASSO, N.; LAWSON, M.; RIVERO, P. A.; TANEJA, A. y VÁZQUEZ, D. A. (2021): El virus de la desigualdad. Cómo recomponer un mundo devastado por el coronavirus a través de una economía equitativa, justa y sostenible. Informe OXFAM 2021. Oxford, OXFAM Intermón. Disponible en https://oxfam.app.box.com/s/m7lab231vgyee3hti2qigu8qvc6o9wd1/file/764560693154 (Consultado 25/01/2021).

CASTRO, T. y SEIZ, M. (2014): “La transformación de las familias en España desde una perspectiva socio-demográfica", en Fundación FOESSA. VII Informe sobre exclusión y desarrollo social en España. Madrid, Fundación FOESSA. Disponible en http://foessa2014.es/informe/ uploaded/documentos_trabajo/13112014045006_7884.pdf (Consultado 10/10/2020).

CASTRO, T.; MARTÍN, T.; CORDERO, J. y SEIZ, M. (2021): Estudios sobre la Economía Española - 2021/04 ¿Cómo mejorar la natalidad en España? Madrid, Fundación de Estudios de Economía Aplicada [FEDEA]. Disponible en https://documentos.fedea.net/pubs/eee/eee202104.pdf (Consultado 25/01/2021).

CENTRO DE INVESTIGACIONES SOCIOLOGÍCAS [CIS] (2014): Opiniones y actitudes sobre la familia (II). Madrid, Centro de Investigaciones Sociológicas. Disponible en http://www.cis.es/cis/export/sites/ default/-Archivos/Marginales/3020_3039/3032/es3032mar.pdf (Consultado 15/10/2020).

COMISION EUROPEA (2020): Plan de recuperación para Europa. Acuerdo sobre el Mecanismo de Recuperación y Resiliencia, Next Generation EU. Disponible en https://ec.europa.eu/info/strategy/recovery-planeurope_es\#contexto (Consultado 21/12/2020).

CONSEJO DE LA JUVENTUD DE ESPAÑA, OBSERVATORIO DE LA EMANCIPACIÓN [OBJOVEM] (2020a): Primer semestre de 2020. Madrid, Consejo de la Juventud de España. Disponible en http://www.cje.org/ descargas/cje7625.pdf (Consultado 10/12/2020). 
CONSEJO DE LA JUVENTUD DE ESPAÑA, OBSERVATORIO DE LA EMANCIPACIÓN [OBJOVEM] (2020b): Juventud en riesgo: análisis de las consecuencias socioeconómicas de la COVID-19 sobre la población joven en España. Madrid, Consejo de la Juventud de España. Disponible en http://www.cje.org/descargas/cje7620.pdf (Consultado 07/06/2021)

CONSEJO ECONOMICO Y SOCIAL (2021): Informe 03/2020 El Sistema de promoción de la autonomía personal y atención a las personas en situación de dependencia. Madrid, Consejo Económico y Social. Disponible en http://www.ces.es/documents/10180/5226728/Inf0320. pdf (Consultado 28/01/2021).

CORTES GENERALES (2021): Resolución de 28 de enero de 2021, del Congreso de los Diputados, por la que se ordena la publicación del Acuerdo de convalidación del Real Decreto-ley 36/2020, de 30 de diciembre, por el que se aprueban medidas urgentes para la modernización de la Administración Pública y para la ejecución del Plan de Recuperación, Transformación y Resiliencia, Boletín Oficial del Estado, 30, p. 12723. Disponible en https://www.boe. es/boe/dias/2021/02/04/pdfs/BOE-A-2021-1613.pdf (Consultado 03/02/2021)

DE LA FUENTE ROBLES, Y. M.; MARTIN, M. C. y ORTEGA, T. (2019): “EI rostro de la desolación. La soledad no elegida en el envejecimiento", en E. Pastor Seller y L. Cano Soriano (eds.), Respuestas del Trabajo Social ante emergencias sociales y problemáticas sociales complejas de México y España. Madrid, Dykinson, pp. 93-114.

DIARIO PÚBLICO (2021/02/01): Una gran mayoría de solicitudes del Ingreso Mínimo Vital se rechazan mientras crece la pobreza severa por la pandemia. Disponible en https://www.publico.es/econo$\mathrm{mia} /$ prestacion-imv-gran-mayoria-solicitudes-ingreso-minimovital-rechazan-crece-pobreza-severa-pandemia.html (Consultado 01/02/2021)

DONAT, M. A, y MARTÍN, M. D. (2020): “Juventud andaluza y emancipación. ¿Independencia residencial y/o autosuficiencia económica?", Papers. Revista de Sociologia, 105 (1), pp. 57-81. doi:http://dx.doi. org/10.5565/rev/papers.2616

DONAT, M. A.; MARTÍN, M. D. y MOLES, E. (2020): “Sustentación principal del hogar en el grupo de edad de 25 a 34 años en España (2006, 2011 y 2015)", Revista Internacional de Sociología, 78 (1): e146. doi. org/10.3989/ris.2020.78.1.18.064.

ESPAÑA (1978): Constitución Española, Boletín Oficial del Estado, 311 de 29 de diciembre. Disponible en https://www.boe.es/buscar/pdf/1978/BOE-A-1978-31229-consolidado.pdf (Consultado $15 / 10 / 2020)$

ESPING-ANDERSEN, G. (coord.) (2013): El déficit de natalidad en Europa: la singularidad del caso español. Barcelona, Obra Social La Caixa.

ESPINOSA, Ma A. y PÉREZ, S. (2021): La infancia vulnerable en España: riesgos y respuestas políticas ante la crisis de la COVID-19. Barcelona, Huygens Editorial.

FUNDACIÓN ADECCO (2019): $8^{\circ}$ Informe \#Monomarentalidad y Empleo. Madrid, Fundación ADECCO. Disponible en https://fundacionadecco.org/wp-content/uploads/2019/10/informe-monomarentalidadempleo-2019-1.pdf (Consultado 15/10/2020).

FUNDACIÓN ADECCO (2020): 9 o Informe \#Monomarentalidad y Empleo. Madrid, Fundación ADECCO. Disponible en https://fundacionadecco.org/wp-content/uploads/2019/10/Descargar-9 \%C2 \%BA-INFORME-MONOPARENTALIDAD-Y-EMPLEO-.pdf (Consultado 15/10/2020).

FUNDACIÓN FOMENTO DE ESTUDIOS SOCIALES Y DE SOCIOLOGÍA APLICADA [FOESSA] (2019): VIII Informe sobre exclusión y desarrollo social en España 2019. Madrid, Cáritas. Disponible en https://caritas-web.s3.amazonaws.com/main-files/uploads/sites/16/2019/05/ Informe-FOESSA-2019-completo.pdf (Consultado 15/10/2020).

FUNDACIÓN THE FAMILY WATCH (2021): X Barómetro de las Familias. Disponible en https://thefamilywatch.org/2021/01/12/2021-x-barometro-de-la-familia/ (Consultado 02/02/2021).
GONZÁLEZ, R. y PASTOR, E. (2019): “Realidades de emergencia social en personas en situación de dependencia: indicadores y respuestas por parte del sistema de Servicios Sociales en España", en E. Pastor y L. Cano (eds.), Respuestas del Trabajo Social ante situaciones de emergencias sociales y problemáticas sociales complejas de México y España. Madrid, Dykinson, pp. 223-240.

INSTITUTO NACIONAL DE ESTADÍSTICA [INE] (2021a): Cifras de población (CP) y estadística de migraciones (EM) de España a 1 de julio de 2020 actualizado a 28 de enero de 2021. Madrid, INE. Disponible https:// www.ine.es/dyngs/INEbase/es/operacion.htm?c=Estadistica_C\&cid $=1254736176951 \&$ menu=ultiDatos\&idp $=1254735572981$ (Consultado 28/01/2021).

INSTITUTO NACIONAL DE ESTADÍSTICA [INE] (2021b): Encuesta Continua de Hogares (ECH) de España 2020. Madrid, INE. Disponible en https://www.ine.es/dyngs/INEbase/es/operacion.htm?c=Estadistic a_C\&cid=1254736176952\&menu=ultiDatos\&idp $=1254735572981$ (Consultado 07/06/2021).

INSTITUTO NACIONAL DE ESTADÍSTICA [INE] (2020a): Brecha de género. Madrid, INE. Disponible en https://www.ine.es/jaxiT3/Datos. htm?t=10883\#!tabs-tabla y https://www.ine.es/ss/Satellite?L=es_ES $\& c=$ INEPublicacion_C\&cid=1259924822888\&p=1254735110672\&p agename=ProductosYServicios \%2FPYSLayout\&param 1=PYSDetal leGratuitas\&param2=1254735350965\&param4=Mostrar (Consultado 28/01/2021).

INSTITUTO NACIONAL DE ESTADÍSTICA [INE] (2020b): Proyección de Hogares 2020-2035. Madrid, INE. Disponible en https://www.ine. es/dyngs/INEbase/es/operacion.htm?c=Estadistica_C\&cid=1254 $736176954 \&$ menu=ultiDatos\&idp $=1254735572981 \quad$ (Consultado 28/01/2021).

INSTITUTO NACIONAL DE ESTADÍSTICA [INE] (2020c): Encuesta de Condiciones de Vida (ECV) 2019. Madrid, INE. Disponible en https://www. ine.es/dyngs/INEbase/es/operacion.htm?c=Estadistica_C\&cid=12 $54736176807 \&$ menu=ultiDatos\&idp $=1254735976608$ (Consultado 28/01/2021).

INSTITUTO NACIONAL DE ESTADÍSTICA [INE] (2019): Encuesta de Fecundidad 2019. Madrid, INE. Disponible en https://www.ine.es/dyngs/ INEbase/es/operacion.htm?c=Estadistica_C\&cid=1254736177006 \&menu=resultados\&idp $=1254735573002 \# !$ tabs-1254736195659 (Consultado 28/01/2021).

JEFATURA DEL ESTADO (2021a). Ley Orgánica 8/2021, de 4 de junio, de protección integral a la infancia y la adolescencia frente a la violencia, Boletín Oficial del Estado, 134, de 5 de junio de 2021, pp. 68657 - 68730. Disponible en https://www.boe.es/boe/dias/2021/06/05/ pdfs/BOE-A-2021-9347.pdf (Consultado 01/06/2021).

JEFATURA DEL ESTADO (2021b): Real Decreto-ley 2/2021, de 26 de enero, de refuerzo y consolidación de medidas sociales en defensa del empleo, Boletín Oficial del Estado, 23, de 27 de enero, pp. 79137954. Disponible en https://boe.es/boe/dias/2021/01/27/pdfs/BOEA-2021-1130.pdf (Consultado 28/01/2021).

JEFATURA DEL ESTADO (2021c): Real Decreto-ley 3/2021, de 2 de febrero, por el que se adoptan medidas para la reducción de la brecha de género y otras materias en los ámbitos de la Seguridad Social y económico, Boletín Oficial del Estado, 29, de 3 de febrero, pp. 12268 12295. Disponible en https://www.boe.es/boe/dias/2021/02/03/ pdfs/BOE-A-2021-1529.pdf (Consultado 04/02/2021).

JEFATURA DEL ESTADO (2020a): Real Decreto-ley 11/2020, de 31 de marzo, por el que se adoptan medidas urgentes complementarias en el ámbito social y económico para hacer frente al COVID-19, Boletín Oficial del Estado, 91, de 01 de abril. Disponible en https://www. boe.es/buscar/pdf/2020/BOE-A-2020-4208-consolidado.pdf (Consultado 15/10/2020).

JEFATURA DEL ESTADO (2020b): Real Decreto-ley 30/2020, de 29 de septiembre, de medidas sociales en defensa del empleo, Boletín Oficial del Estado, 259, de 30 de septiembre. 82169-82211 Disponible en https://www.boe.es/boe/dias/2020/09/30/pdfs/BOE-A-2020-11416. pdf (Consultado 10/10/2020). 
JEFATURA DEL ESTADO (2020c): Real Decreto-ley 37/2020, de 22 de diciembre, de medidas urgentes para hacer frente a las situaciones de vulnerabilidad social y económica en el ámbito de la vivienda y en materia de transportes, Boletín Oficial del Estado, 334, de 23 de diciembre. Disponible en https://www.boe.es/buscar/pdf/2020/BOEA-2020-16824-consolidado.pdf (Consultado 15/01/2021).

JEFATURA DEL ESTADO (2020d): Real Decreto-ley 20/2020, de 29 de mayo, por el que se establece el ingreso mínimo vital, Boletín Oficial del Estado, 154, pp. 36022-36065. Disponible en https://www.boe. es/eli/es/rdl/2020/05/29/20/dof/spa/pdf (Consultado 28/10/2020).

JEFATURA DEL ESTADO (2020e): Real Decreto-ley 36/2020, de 30 de diciembre, por el que se aprueban medidas urgentes para la modernización de la Administración Pública y para la ejecución del Plan de Recuperación, Transformación y Resiliencia, Boletín Oficial del Estado, 341, de 31 de diciembre. Disponible en https://www.boe. es/buscar/pdf/2020/BOE-A-2020-17340-consolidado.pdf (Consultado 10/10/2021). Corrección de errores publicado en el Boletín Oficial del Estado, 22, de 26 de enero de 2021, p. 7325. Disponible en https://www.boe.es/boe/dias/2021/01/26/pdfs/BOE-A-2021-1064. pdf (Consultado 28/01/2021).

JEFATURA DEL ESTADO (2019): Real Decreto-ley 6/2019, de 1 de marzo, de medidas urgentes para garantía de la igualdad de trato y de oportunidades entre mujeres y hombres en el empleo y la ocupación, Boletín Oficial del Estado, 57, pp. 21692-21717. Disponible en https://www.boe.es/buscar/doc.php?id=BOE-A-2019-3244 (Consultado $10 / 11 / 2020)$.

LÓPEZ OLLER, J. (2020): Juventud en riesgo. Análisis de las consecuencias socioeconómicas de la COVID-19 sobre la población joven en España. Madrid, Instituto de la Juventud. Disponible en http://www.cje. org/descargas/cje7620.pdf (Consultado 10/01/2021).

LÓPEZ PELÁEZ, A. y CASTILLO DE MESA, J. (2020): “El Trabajo Social con familias en la época del big data. Nuevas estrategias de intervención en el siglo XXI", en E. Pastor-Seller, J. Ferreira, M.D. Dores Guerreiro y F. Chacon (eds.), Familias, identidades y cambio social en España y Portugal. Pamplona, Aranzadi-Thomson Reuteres, pp. 107-118.

LÓPEZ PELÁEZ, A. y SEGADO, S. (2013): “Empowerment, WeU-Being and the Welfare State: Family Social Work in Spain", en A. Moreno (ed.), Family Well-Being. European Perspectives. New York, Springer, pp. 277-302.

MALGESINI, G. (2019): Estudio sobre las familias monoparentales perceptores de rentas mínimas. Madrid, Red Europea de Lucha contra la pobreza [EAPN]. Disponible en https://www.eapn.es/ARCHIVO/ documentos/documentos/1568715475_estudio-familias-monoparentales.pdf (Consultado 10/12/2020).

MARCALETTI, F. y CAVALLOTTI, R. (2021): “Un estudio exploratorio sobre capital social familiar en España y sus relaciones con otras formas de capital social", Revista Española de Investigaciones Sociológicas, 173, pp. 47-68. http://dx.doi.org/10.5477/cis/reis.173.47

MARTíN, J. M.; SUSO, A.; FERNÁNDEZ, J.; MACÍAS, I. y CONTRERAS, L. (2021): Superar la pandemia y reducir la desigualdad. Como hacer frente a la crisis sin repetir errores. Madrid, OXFMAN Intermón. Disponible en https://f.hubspotusercontent20.net/hubfs/426027/ Oxfam-Website/oi-informes/superar-covid-reducir-desigualdadoxfam-intermon.pdf (Consultado 25/01/2021).

MINISTERIO DE POLÍTICA TERRITORIAL Y FUNCIÓN PÚBLICA (2020): Real Decreto 452/2020, de 10 de marzo, por el que se desarrolla la estructura orgánica básica del Ministerio de Derechos Sociales y Agenda 2030, y se modifica el Real Decreto 139/2020, de 28 de enero, por el que se establece la estructura orgánica básica de los departamentos ministeriales, Boletín Oficial del Estado, 63, de 12 de marzo, pp. 24611-24625. Disponible en https://www.boe. es/boe/dias/2020/03/12/pdfs/BOE-A-2020-3512.pdf (Consultado 25/10/2020).

MINISTERIO DE SANIDAD, CONSUMO Y BIENESTAR SOCIAL (2013): Catálogo de Referencia de Servicios Sociales. Madrid, Ministerio de Sanidad, Consumo y Bienestar Social. Disponible en https://www.
mscbs.gob.es/ssi/familiasInfancia/ServiciosSociales/docs/CatalogoServiciosSociales.pdf (Consultado 28/09/2020).

MINISTERIO DE SANIDAD, CONSUMO Y BIENESTAR SOCIAL (2015): Plan Integral de Apoyo a la Familia 2015-2017. Madrid, Dirección General de Servicios para las Familias y la Infancia. Disponible en https:// www.mscbs.gob.es/ssi/familias/nfancia/ayudas/docs2013-14/ PIAF20152017.pdf (Consultado 28/09/2020).

MINISTERIO DE DERECHOS SOCIALES Y AGENDA 2030 (2020a): Informe de Rentas Mínimas de Inserción 2019. Madrid, Dirección General de Servicios para las Familias y la Infancia. Disponible en https:// www.mscbs.gob.es/ssi/familiasInfancia/ServiciosSociales/RentasMinimas/Informe_RRMM_2019_Provisional.pdf (Consultado 15/10/2020).

MINISTERIO DE DERECHOS SOCIALES Y AGENDA 2030 (2020b): Orden DSA/789/2020, de 27 de julio, por la que se publica el Acuerdo del Consejo de Ministros de 14 de julio de 2020, por el que se formalizan los criterios de distribución y la distribución resultante de los créditos acordados por el Consejo Territorial de Servicios Sociales y del Sistema para la Autonomía y Atención a la Dependencia, que se destinan a la financiación de los planes o programas sociales, para el ejercicio 2020, Boletín Oficial del Estado, 218, de 13 de agosto de 2020, pp. 69287-69296. Disponible en https://www.mscbs.gob.es/ ssi/familiasInfancia/ServiciosSociales/docs/BOE-A-2020-9656.pdf (Consultado 07/06/2021).

MINISTERIO DE DERECHOS SOCIALES Y AGENDA 2030 (2021): Guía de ayudas sociales y servicios para las familias 2021. Madrid, Dirección General de Diversidad Familiar y Servicios Sociales. Disponible en https://www.mscbs.gob.es/ssi/familiasInfancia/pdf/ Guia_ayudas_y_servicios_para_familias_2021.pdf (Consultado 07/06/2021).

MINISTERIO DE SANIDAD, CONSUMO Y BIENESTAR SOCIAL (2019a): Informe de Rentas Mínimas de Inserción 2018. Madrid, Dirección General de Servicios para las Familias y la Infancia. Disponible en https:// www.mscbs.gob.es/ssi/familiasInfancia/ServiciosSociales/RentasMinimas/Informe_2018.pdf (Consultado 12/10/2020).

MINISTERIO DE SANIDAD, CONSUMO Y BIENESTAR SOCIAL (2019b): Estrategia Nacional de Prevención y Lucha contra la Pobreza y la EXclusión Social 2019-2023. Madrid, Ministerio de Sanidad, Consumo y Bienestar Social. Disponible en https://www.mscbs.gob.es/ssi/ familiasInfancia/inclusionSocial/inclusionSocialEspana/Estrategia Prev_LuchaPobreza_19-23.pdf (Consultado 20/12/2020).

MINISTERIO DE TRANSPORTES, MOVILIDAD Y AGENDA URBANA (2018): Plan Estatal de Vivienda (2018-2021). Madrid, Ministerio de Transportes, Movilidad y Agenda Urbana. Disponible en https://www. mitma.gob.es/arquitectura-vivienda-y-suelo/programas-de-ayudas-a-la-vivienda (Consultado 21/12/2020).

MORENO, A. (ed.) (2013): Family Well-Being. European Perspectives. New York, Springer.

MORENO, A. (2000): “Las familias monoparentales en España”, Revista Internacional de Sociología, 26, pp. 39-63.

MORENO, A. (coord.); LÓPEZ, A. y SEGADO, S. (2012): La transición de los jóvenes a la vida adulta. Crisis económica y emancipación tardía. Barcelona, Obra Social "La Caixa”.

MORENO, A; ORTEGA, M. Y GAMERO, C. (2017): “Los modelos familiares en España: reflexionando sobre la ambivalencia familiar desde una aproximación teórica", Revista Española de Sociología, 26 (2), pp. 149-167 https://doi.org/10.22325/fes/res.2016.5.

MORENO, A. y VICENTE, J. A. (2016): “Situación Familiar, Protección Social y Bienestar de las Personas Mayores en Perspectiva Europea Comparada", Research on Ageing and Social Policy, 4 (2), pp. 96-127. doi: 10.17583/rasp.2016.2048.

NACIONES UNIDAS (2020a): Familias en desarrollo: Copenhague + 25. Disponible en https://www.mscbs.gob.es/ssi/familiaslnfancia/diainternacioanalfamilias/doc/Copenhague.pdf (Consultado 28/01/2021) 
NACIONES UNIDAS (2020b): The Sustainable Development Goals Report 2020. Disponible en https://unstats.un.org/sdgs/report/2020/\#sdggoals (Consultado 15/01/2021).

NACIONES UNIDAS (2019a): Aplicación de los objetivos del Año Internacional de la Familia y sus procesos de seguimiento. Disponible en https://undocs.org/es/A/75/61 \%E2 \%80 \%93E/2020/4 (Consultado 15/01/2021).

NACIONES UNIDAS (2019b): El progreso de las mujeres en el mundo 20192020: Familias en un mundo cambiante, ONU-Mujeres, Nueva York. Disponible en https://www.unwomen.org/es/news/stories/2019/6/ press-release-progress-of-the-worlds-women-2019\#www.progress. unwomen.org (Consultado 10/01/2021).

NACIONES UNIDAS (2015): Resolución aprobada por la Asamblea General el 25 de septiembre de 2015: 70/1. Transformar nuestro mundo: la Agenda 2030 para el Desarrollo Sostenible. A/RES/70/1. Disponible en https://www.agenda2030.gob.es/sites/default/files/recursos/APROBACI \%C3 \%93N \%20AGENDA \%202030.pdf (Consultado 28/10/2020).

OXFAM-Intermón (2019): Informe Desigualdad 1-Igualdad de Oportunidades 0 . La inmovilidad social y la condena de la pobreza. Madrid, OXFMAN Intermón. Disponible en https://www.oxfamintermon.org/ es/inmovilidad-social-condena-pobreza https://web.oxfamintermon.org/sites/default/files/documentos/files/desigualdad-1-igualdad-oportunidades-0.pdf (Consultado 28/10/2020).

PARLAMENTO EUROPEO (2020): Regulation (EU) 2020/559 of the European Parliament and of the Council of 23 April 2020 amending Regulation (EU) No 223/2014 as regards the introduction of specific measures for addressing the outbreak of COVID-19. Official Journal of the European Union de 24 de abril de 2020. Disponible en https:// eur-lex.europa.eu/legal-content/ES/TXT/PDF/?uri=CELEX:32020R05 59\&from=EN (Consultado 20/12/2020).

PARLAMENTO EUROPEO, CONSEJO DE LA UNIÓN EUROPEA Y COMISIÓN EUROPEA (2000): Carta de los derechos fundamentales de la Unión Europea, Diario Oficial de las Comunidades Europeas, 364 de 18/12/2000. Disponible en https://www.europarl.europa.eu/charter/pdf/text_es.pdf (Consultado 28/10/2020).

PASTOR, E. (2017): "Mechanisms for participation in the public system of social services in Spain: opportunities for the development of social work with a citizenist approach", European Journal of Social Work, 20 (3), pp. 441-458 DOI: 10.1080/13691457.2017.1283588.

PASTOR, E.; FERREIRA, J.; GUERREIRO, M. D. y CHACON, F. (eds.) (2020): Familias, identidades y cambio social en España y Portugal. Pamplona, Aranzadi-Thomson Reuteres.

PASTOR, E. y SÁNCHEZ, I. C. (2020): “Atención a las familias e infancia en riesgo social", Interacción y Perspectiva, 10 (1), pp. 29-46.

PASTOR, E., VERDE, C. y LIMA, A.I. (2019): "Impact of neo-liberalism in Spain: research from social work in relation to the public system of social services", European Journal of Social Work, 22 (2), pp. 277-288, DOI:10.1080/13691457.2018.1529663.

PRESIDENCIA DEL GOBIERNO (2021): Plan de recuperación, transformación y resiliencia. https://www.lamoncloa.gob.es/temas/fondos-recuperacion/Documents/30042021-Plan_Recuperacion_ \%20Transformacion_\%20Resiliencia.pdf (Consultado 07/06/2021).

SASTRE, A. (2015): Más solas que nunca. La pobreza infantil en familias monomarentales. Madrid, Save the children.

VERDE, C.; GONZÁLEZ, R.; VÁZQUEZ, R. y MORENO, L. V. (2020): “¿Política social para "la familia" o para las familias en España? El desajuste con la diversidad familiar, con especial referencia a las familias "reconstituidas", en E. Pastor, J. Ferreira, M. D. Dores Guerreiro y F. Chacon (eds.), Familias, identidades y cambio social en España y Portugal. Pamplona, Aranzadi-Thomson Reuteres, pp. 83-103. 